\title{
Infinitely Divisible Representations and Positive Definite Functions on a Compact Group
}

\author{
K. R. PARTHASARATHY \\ Department of Mathematics, Statistical Laboratory \\ University of Manchester
}

Received September 9, 1969

\begin{abstract}
A complete description of the infinitely divisible positive definite functions on a compact group is given. Their relation to infinitely divisible representations is also discussed.
\end{abstract}

\section{§ 1. Introduction}

In certain problems of quantum field theory it is necessary to define the notion of a continuous tensor product of Hilbert spaces. In his attempt to construct such tensor products Streater [3] has introduced the notion of an infinitely divisible group representation. These in turn are described by infinitely divisible positive definite functions on the group. If the group is abelian such positive definite functions are in one to one correspondence with the so called infinitely divisible distributions on the character group. The reader may find a detailed account of these in [1]. Here we shall give a complete description of the infinitely divisible positive definite functions on a compact group and describe some of the infinitely divisible representations.

\section{§ 2. Infinitely Divisible Group Representations and Positive Definite Functions}

Throughout this paper we shall denote by $G$ a fixed compact metric group. By a representation of $G$ we mean a continuous unitary representation of $G$ in a complex separable Hilbert space with an inner product (.,.). Let $T^{(k)}, k=1,2, \ldots$ be an enumeration of all the inquivalent irreducible representations (up to equivalence). Suppose $T^{(k)}$ operates in a Hilbert space $V_{k}$ of dimension $d_{k}$. Let $\varepsilon_{1}^{k}, \varepsilon_{2}^{k}, \ldots, \varepsilon_{d_{k}}^{k}$ be a 
fixed orthonormal basis in $V_{k}$ and let

$$
\psi_{r s}^{(k)}(g)=\left(T_{g}^{(k)} \varepsilon_{r}^{k}, \varepsilon_{s}^{k}\right), \quad 1 \leqq r, s \leqq d_{k} .
$$

Integration in $G$ will always be with respect to the normalised Haar measure in G. Under these assumptions it is well known (cf. [2]) that the functions $\psi_{r s}^{(k)}$ constitute an orthogonal basis for the Hilbert space $L_{2}(G)$ and

$$
\int\left|\psi_{r s}^{(k)}(g)\right|^{2} d g=\frac{1}{d_{k}}, \quad 1 \leqq r, s \leqq d_{k}, \quad k=1,2, \ldots
$$

Let $\mathscr{I}$ denote the multiplicative group of complex numbers of modulus unity and $D$ be the group of all continuous homomorphisms of $G$ into $\mathscr{I}$. With the uniform topology, $D$ becomes a discrete group with a finite or countable number of elements.

Definition 2.1. A representation $U$ of $G$ with cyclic vector $x$ in a Hilbert space $\mathscr{H}$ is said to be infinitely divisible if, for any positive integer $q$, there exists a representation $U^{1 / q}$ with cyclic vector $x^{1 / q}$ in a Hilbert space $\mathscr{H}^{1 / q}$ such that $U$ is unitarily equivalent to the representation $\underbrace{U^{1 / q} \otimes \cdots \otimes U^{1 / q}}_{q \text { factors }}$ restricted to the cyclic subspace generated by $x^{1 / q} \otimes x^{1 / q} \otimes \cdots \otimes x^{1 / q}$ by the action of the group. Under this equivalence $x$ and $x^{1 / q} \otimes \cdots \otimes x^{1 / q}$ are to correspond.

Definition 2.2. A function $\phi$ defined on $G$ is said to be positive definite if and only if it is continuous and for every finite collection of points $g_{1}, g_{2}, \ldots, g_{n}$ in $G$ and complex numbers $a_{1}, a_{2}, \ldots, a_{n}$, the following inequality holds: $\sum a_{i} \bar{a}_{j} \phi\left(g_{i} g_{j}^{-1}\right) \geqq 0$. Equivalently, a function $\phi$ is positive definite if and only if it is continuous and for every complex valued bounded measurable function $f$ on $G, \int f(g) \overline{f(h)} \phi\left(g h^{-1}\right) d g d h \geqq 0$.

Definition 2.3. A positive definite function $\phi$ is said to be infinitely divisible if, for any positive integer $q$, there exists a positive definite function $\phi_{q}$ such that $\phi(g)=\left[\phi_{q}(g)\right]^{q}$ for all $g \in G$.

We shall say that a positive definite function $\phi$ is normalised if $\phi(e)=1$ where $e$ is the identity element of $G$. Let $\mathscr{P}$ be the space of all normalized positive definite functions on $G$ with the uniform topology. If $U$ is a representation of $G$ in Hilbert space $\mathscr{H}$ and $x \in \mathscr{H}$ is a unit vector, then the function $\left(U_{g} x, x\right)$ is in $\mathscr{P}$. Conversely every element of $\mathscr{P}$ arises in this manner. The vector $x$ can be assumed to be cyclic without loss of generality. It is an easy consequence of Definition 2.1 and 2.2 that every infinitely divisible positive definite function on $G$ arises from an infinitely divisible representation and vice versa. Thus the problem of finding the infinitely divisible representations of $G$ reduces to the problem of finding the infinitely divisible positive definite functions of $G$. 


\section{§ 3. Some Lemmas on Positive Definite Functions}

Lemma 3.1. A complex valued function $\phi$ defined on $G$ belongs to $\mathscr{P}$ if and only if it is of the form

$$
\phi(g)=\sum_{k} p_{k}\left\{\sum_{1 \leqq r, s \leqq d_{k}} a_{r s}^{(k)} \psi_{r s}^{(k)}(g)\right\}
$$

where $p_{1}, p_{2}, \ldots$ are non negative constants such that $\sum_{k} p_{k}=1,\left(\left(a_{r s}^{(k)}\right)\right)$ is a positive definite constant matrix of order $d_{k} \times d_{k}$ and trace unity for every $k$, $\psi_{r s}^{(k)}$ are the functions defined by (2.1) and the convergence of the above series is uniform.

Proof. The function $\phi \in \mathscr{P}$ if and only if there exists a representation $U$ and unit vector $x$ such that $\phi(g)=\left(U_{g} x, x\right)$. $U$ can be decomposed into a direct sum of primary representations. Each primary representation is a finite or countable direct sum of copies of some $T^{(k)}$. The lemma is an immediate consequence of these facts.

Lemma 3.2. Suppose $\phi_{n} \in \mathscr{P}, n=0,1,2, \ldots$, and $\phi_{n}(g) \rightarrow \phi_{0}(g)$ as $n \rightarrow \infty$ for every $g$. Then $\phi_{n} \rightarrow \phi_{0}$ uniformly in $G$.

Proof. By Lemma 3.1 we can write

$$
\phi_{n}(g)=\sum_{k} p_{n k} \sum_{1 \leqq r, s \leqq d_{k}} a_{r s}^{(k)}(n) \psi_{r s}^{(k)}(g), \quad n=0,1,2 \ldots
$$

where, for each fixed $n, p_{n k}$ are non negative numbers such that $\sum_{k} p_{n k}=1$ and for each fixed $n$ and $k$, the matrix $\left(\left(a_{r s}^{(k)}(n)\right)\right)$ is positive definite and of trace unity. Since $\left|\phi_{n}(g)\right| \leqq 1$ and $\phi_{n}(g) \rightarrow \phi_{0}(g)$ as $n \rightarrow \infty$ for every $g$, we have

$$
\lim _{n \rightarrow \infty} \int \phi_{n}(g) \overline{\psi_{r s}^{(k)}(g)} d g=\int \phi_{0}(g) \overline{\psi_{r s}^{(k)}(g)} d g .
$$

Since the functions $\psi_{r s}^{(k)}(g)$ are mutually orthogonal in $L_{2}(G)$ and have norm $d_{k}^{-\frac{1}{2}}(\operatorname{see}(2.2))$ we have

$$
\lim _{n \rightarrow \infty} p_{n k} a_{r s}^{(k)}(n)=p_{0 k} a_{r s}^{(k)}(0) \text { for every } r, s, k .
$$

Since $\sum_{r=1}^{d_{k}} a_{r r}^{(k)}=1$, we obtain

$$
\begin{aligned}
& \lim _{n \rightarrow \infty} p_{n k}=p_{0 k}, \\
& \lim _{n \rightarrow \infty} a_{r s}^{(k)}(n)=a_{r s}^{(k)}(0) \text { if } p_{0 k} \neq 0 .
\end{aligned}
$$


For each fixed $n,\left\{p_{n 1}, p_{n 2} \ldots\right\}$ can be viewed upon as masses of a discrete probability distribution. Hence (3.1) implies that ${ }^{1}$

$$
\lim _{n \rightarrow \infty} \sum_{k}\left|p_{n k}-p_{0 k}\right|=0 \text {. }
$$

Since $\sum_{r, s} a_{r s}^{(k)} \psi_{r s}^{(k)} \in \mathscr{P}$, its absolute value is not greater than unity. This together with (3.3) implies that $\phi_{n} \rightarrow \phi_{0}$ uniformly. This completes the proof of the lemma.

Lemma 3.3. Let $\phi_{n} \in \mathscr{P}$ be a sequence such that $\operatorname{Re} \phi_{n}$ (i.e. the real part of $\left.\phi_{n}\right)$ converges uniformly. Then the sequence $\left\{\phi_{n}\right\}$ is conditionally compact in the uniform topology.

Proof. Suppose $\phi \in \mathscr{P}$ and $g, h$ are two points in $G$. Let $U$ be a representation and $x$ be a unit vector such that $\phi(g) \equiv\left(U_{g} x, x\right)$. We have

$$
\begin{aligned}
|\phi(g)-\phi(h)| & =\left|\left(U_{g} x, x\right)-\left(U_{h} x, x\right)\right| \\
& \leqq\left\|U_{g} x-U_{h} x\right\| \\
& \leqq\left\|U_{g^{-1} h} x-x\right\| \\
& \leqq\left\{2\left[1-\operatorname{Re} \phi\left(g^{-1} h\right)\right]\right\}^{\frac{1}{2}} .
\end{aligned}
$$

If $\operatorname{Re} \phi_{n}$ converges uniformly it follows from the above inequality that the sequence $\left\{\phi_{n}\right\}$ is uniformly equicontinuous. An application of Arzela-Ascoli theorem completes the proof of the lemma.

Lemma 3.4. Let $\psi$ be a continuous function defined on $G$ and satisfying the inequality

$$
\int_{G \times G} f(g) \overline{f(h)} \psi\left(g h^{-1}\right) d g d h \geqq 0
$$

for every bounded measurable function $f$ such that $\int f(g) d g=0$. Then $\psi(g)-\int \psi(g) d g$ is positive definite.

Proof. Let $f$ be a bounded measurable function such that $\int f(g) d g=m$. Let $\int \psi(g) d g=a$. Then the invariance of the Haar measure implies that

$$
\begin{gathered}
\int f(g) \overline{f(h)}\left[\psi\left(g h^{-1}\right)-a\right] d g d h \\
=\int(f(g)-m)(\overline{f(h)}-\bar{m}) \psi\left(g h^{-1}\right) d g d h \geqq 0 .
\end{gathered}
$$

This implies that $\psi(g)-a$ is positive definite. The proof of the lemma is complete.

Lemma 3.5. For every positive definite function $\phi$ on $G$,

$$
\int \operatorname{Im} \phi(g) d g=0 .
$$

${ }^{1}$ For a proof of this the reader may refer to Lemma 9.1, page 206 in [1]. 
Proof. This is an immediate consequence of the fact that $\phi\left(g^{-1}\right)=\overline{\phi(g)}$ and the Haar measure is invariant under the mapping $g \rightarrow g^{-1}$.

Lemma 3.6. For any $\phi \in \mathscr{P}, g_{1}, g_{2} \in G$,

$$
\left[1-\operatorname{Re} \phi\left(g_{1} g_{2}\right)\right]^{\frac{1}{2}} \leqq\left[1-\operatorname{Re} \phi\left(g_{1}\right)\right]^{\frac{1}{2}}+\left[1-\operatorname{Re} \phi\left(g_{2}\right)\right]^{\frac{1}{2}} .
$$

Proof. Let $U$ be a representation in a Hilbert space and $x$ a unit vector such that $\phi(g)=\left(U_{g} x, x\right)$ for all $g \in G$. Then

$$
\left\|U_{g} x-x\right\|=[2(1-\operatorname{Re} \phi(g))]^{\frac{1}{2}} .
$$

Now we have

$$
\begin{aligned}
\left\|U_{g_{1} g_{2}} x-x\right\| & \leqq\left\|U_{g_{1} g_{2}} x-U_{g_{1}} x\right\|+\left\|U_{g_{1}} x-x\right\| \\
& \leqq\left\|U_{g_{2}} x-x\right\|+\left\|U_{g_{1}} x-x\right\|
\end{aligned}
$$

(3.4) and (3.5) imply the required inequality. This completes the proof of the lemma.

Lemma 3.7. Let $H \subset G$ be a both open closed subgroup of G. Suppose $\psi$ is a positive definite function defined on $H$. Let $\phi$ be defined by

$$
\begin{aligned}
\phi(g) & =\psi(g) & \text { if } & & g \in H, \\
& =0 & \text { if } & & g \notin H .
\end{aligned}
$$

Then $\phi$ is a positive definite function on $G$.

Proof. The continuity of $\phi$ is obvious. Let now $a_{1}, a_{2}, \ldots, a_{n}$ be any $n$ complex numbers and $g_{1}, g_{2}, \ldots, g_{n}$ any $n$ points in $G$. Each element $g_{i}$ belongs to some left coset of $H$ in $G$. Let $H_{i}=H w_{i}, i=1,2, \ldots, k$ be the distinct cosets to which $g_{1}, g_{2}, \ldots, g_{n}$ belong. Then

$$
\begin{gathered}
\sum_{1 \leqq i, j \leqq n} a_{i} \bar{a}_{j} \phi\left(g_{i} g_{j}^{-1}\right)=\sum_{i}\left\{\sum_{(r, s): g_{r}, g_{s} \in H_{i}} a_{r} \overline{a_{s}} \phi\left(g_{r} g_{s}^{-1}\right)\right\} \\
=\sum_{i=1}^{k}\left\{\sum_{(r, s): g_{r}, g_{s} \in H_{i}} a_{r} \overline{a_{s}} \phi\left(\left(g_{r} w_{i}^{-1}\right)\left(g_{s} w_{i}^{-1}\right)^{-1}\right)\right\} .
\end{gathered}
$$

Since $g_{r} w_{i}^{-1} \in H$ for all $r$ such that $g_{r} \in H w_{i}$ and $\phi=\psi$ on $H$, it follows that every term within brackets on the right hand side of the above equation is non negative. This completes the proof of the lemma.

\section{§ 4. Representation of Infinitely Divisible Positive Definite Functions}

Before proceeding to the statement of the main result we shall prove a lemma on the infinitely divisible elements of $\mathscr{P}$.

Lemma 4.1. Let $\phi \in \mathscr{P}$ be infinitely divisible and $\phi(g) \neq 0$ for every $g \in G$. Suppose $\phi=\phi_{n}^{n}, n=1,2, \ldots$ where $\phi_{n} \in \mathscr{P}$ for all $n$. Then there exists a sequence $\chi_{n} \in D$ such that $\phi_{n} \chi_{n}$ converges uniformly to unity. 
Proof. Since $\phi=\phi_{n}^{n}$ for all $n$, it follows that $n\left(\left|\phi_{n}\right|-1\right)$ converges uniformly to $\log |\phi|$ as $n \rightarrow \infty$. By Lemma 3.1 we may assume that $\phi_{n}$ is given by

$$
\phi_{n}(g)=\sum_{k=1}^{\infty} p_{n k} \sum_{1 \leqq r, s \leqq d_{k}} a_{r s}^{(k)}(n) \psi_{r s}^{(k)}(g) .
$$

Since $\int\left|\phi_{n}(g)\right|^{2} d g \rightarrow 1$ as $n \rightarrow \infty$, we have from (2.2)

$$
\lim _{n \rightarrow \infty} \sum_{k} p_{n k}^{2} \sum_{1 \leqq r, s \leqq d_{k}} \frac{\left|a_{r s}^{(k)}(n)\right|^{2}}{d_{k}}=1 .
$$

Since, for each $k$ and $n, a_{r s}^{(k)}$ is a positive definite matrix of trace unity and $\sum_{k} p_{n k}=1$, it follows that

$$
\lim _{n \rightarrow \infty} \sum p_{n k}^{2} / d_{k}=1
$$

Hence

Hence

Thus

$$
\begin{aligned}
& \lim _{n \rightarrow \infty} \sum_{k: d_{k}=1} p_{n k}^{2}+\frac{1}{2} \sum_{k: d_{k} \geqq 2} p_{n k}^{2}=1, \\
& \lim _{n \rightarrow \infty} \sum_{k: d_{k}=1} p_{n k}^{2}+\sum_{k: d_{k} \geqq 2} p_{n k}^{2}=1 .
\end{aligned}
$$

$$
\lim _{n \rightarrow \infty} \sum_{k: d_{k}=1} p_{n k}^{2}=1 \text {. }
$$

$$
\lim _{n \rightarrow \infty}\left(\sup _{k: d_{k}=1} p_{n k}\right)\left(\sum_{k: d_{k}=1} p_{n k}\right)=1 \text {. }
$$

In other words there exists a sequence $k_{n}$ such that $\lim _{n \rightarrow \infty} p_{n k n}=1$, where $T^{\left(k_{n}\right)}$ is a one dimensional representation of $G$. Let $\chi_{n}$ be the corresponding homomorphism of $G$ into $\mathscr{I}$. Then

$$
\phi_{n}(g) \bar{\chi}_{n}(g)=p_{n k_{n}}+\gamma_{n}(g)
$$

where $\gamma_{n}(g)$ is also positive definite and $\gamma_{n}(e)=1-p_{n k_{n}}$. Since $\gamma_{n}(e) \rightarrow 0$ as $n \rightarrow \infty$ and $\left|\gamma_{n}(g)\right| \leqq \gamma_{n}(e)$, it follows that $\phi_{n} \bar{\chi}_{n}$ converges uniformly to unity. This completes the proof of the lemma.

Theorem 4.1. Let $\phi$ be a normalised infinitely divisible positive definite function defined on $G$. Suppose $\phi(g) \neq 0$ for every $g \in G$. Then $\phi$ admits a representation

$$
\phi(g)=\chi(g) \exp [\gamma(g)-\gamma(e)] \text { for all } g \in G,
$$

where $\gamma$ is a positive definite function on $G$ and $\chi$ is an infinitely divisible element of the group of homomorphisms of $G$ into $\mathscr{I}$.

Conversely every function $\phi$ of the form given by (4.1) is infinitely divisible and without zeros. 
Proof. The converse part is trivial. In order to prove the first part choose a sequence $\phi_{n} \in \mathscr{P}$ such that $\phi_{n}^{n}=\phi$. By Lemma 4.1, there exists a $\chi_{n} \in D$ such that $\phi_{n} \chi_{n} \rightarrow 1$ uniformly as $n \rightarrow \infty$. Let $\psi_{n}=\phi_{n} \chi_{n}$. Then $\phi=\psi_{n}^{n} \bar{\chi}_{n}^{n}$. Let $g$ be any point in $G$ and $A_{g}$ be the closed abelian subgroup generated by $g$. The functions $\psi_{n}$ and $\phi$ restricted to $A_{g}$ are Fourier transforms of probability measures $\mu_{n}$ and $\mu$ respectively on the character group $\hat{A}_{g}$ of $A_{g}$. Since $\psi_{n} \rightarrow 1$ uniformly $\mu_{n}$ converges weakly to the measure degenerate at the identity of $\hat{A}_{g}$. Further $\mu_{n}^{* n}$ is a translate of $\mu$ in $A_{g}$. Hence by Theorem 5.1, page 89, [1], it follows that

Hence

$$
\lim _{n \rightarrow \infty}\left|\psi_{n}(g)^{n}-\exp n\left[\psi_{n}(g)-1\right]\right|=0 .
$$

$$
\lim _{n \rightarrow \infty}\left|\phi(g)-\bar{\chi}_{n}(g)^{n} \exp n\left[\psi_{n}(g)-1\right]\right|=0 .
$$

Since (4.2) holds for every $g$, Lemma 3.2 implies that the convergence is uniform. Since $\phi$ has no zeros we have

$$
\lim _{n \rightarrow \infty} \sup _{g}\left|n\left[\operatorname{Re} \psi_{n}(g)-1\right]-\log \right| \phi(g)||=0 .
$$

By Lemma 3.5,

Let

$$
n \int\left(\psi_{n}(g)-1\right) d g=n \int\left(\operatorname{Re} \psi_{n}(g)-1\right) d g=a_{n}, \quad \text { say. }
$$

$$
a=\int \log |\phi(g)| d g .
$$

By (4.3), $a_{n} \rightarrow a$ as $n \rightarrow \infty$. Since $\psi_{n}(g)$ is positive definite $n\left[\psi_{n}(g)-1\right]$ satisfies the conditions of Lemma 3.4. Hence the function $\gamma_{n}$ defined by

$$
\gamma_{n}(g)=n\left[\psi_{n}(g)-1\right]-a_{n}
$$

is positive definite. By (4.3), $\operatorname{Re} \gamma_{n}(g)$ converges uniformly to $\log |\phi(g)|-a$. By Lemma 3.3, the sequence $\gamma_{n}$ is conditionally compact in the uniform topology. Now (4.2) implies that $\bar{\chi}_{n}^{n}$ is also conditionally compact in the uniform topology. Since the group $D$ is discrete, it follows that the set $\left\{\bar{\chi}_{n}^{n}\right\}$ is finite. Hence there exists a $\chi \in D$ such that $\chi=\bar{\chi}_{n !}^{n !}$ for infinitely many $n$. This $\chi$ is infinitely divisible. Now extracting a suitable convergent subsequence from $\gamma_{n !}$ and using (4.2) we conclude that

$$
\phi(g)=\chi(g) \exp (\gamma(g)+a)
$$

where $\gamma$ is positive definite. Since $\phi(e)=\chi(e)=1$, it follows that $a=-\gamma(e)$. This completes the proof of the theorem.

Now we shall proceed to analyse the nature of infinitely divisible positive definite functions with zeros.

Lemma 4.2. Let $\phi \in \mathscr{P}$ be infinitely divisible. Then the set $\{g: \phi(g) \neq 0\}$ is a both open closed subgroup of $G$. 
Proof. Let $\left\{\phi_{n}\right\}$ be a sequence of elements on $\mathscr{P}$ such that $\phi=\phi_{n}^{n}$. In particular $|\phi|^{2}=\left|\phi_{n}\right|^{2 n}$ and $|\phi(g)|^{2} \neq 0$ for some $g$ if and only if $\varlimsup_{n \rightarrow \infty} n\left(1-\left|\phi_{n}(g)\right|^{2}\right)<\infty$. It follows from Lemma 3.6 that the set $\left\{g: \varlimsup_{n \rightarrow \infty} n\left(1-\left|\phi_{n}(g)\right|^{2}\right)<\infty\right\}$ is actually a subgroup. Since $\phi(g)$ is continuous this subgroup is open. Since an open subgroup is closed the proof of the lemma is complete.

Theorem 4.2. A normalised positive definite function $\phi$ on $G$ is infinitely divisible if and only if it admits a representation

$$
\begin{aligned}
\phi(g) & =\chi(g) \exp [\gamma(g)-\gamma(e)] \text { if } g \in H \\
& =0 \text { otherwise }
\end{aligned}
$$

where $H$ is a both open closed subgroup of $G, \chi$ is an infinitely divisible homomorphism of $H$ into $\mathscr{I}$ and $\gamma$ is a positive definite function on $H$.

Proof. This is an immediate consequence of Theorem 4.1, Lemmas 4.2 and 3.7.

Remark 1. There are many compact groups which do not possess an infinitely divisible homomorphism into $\mathscr{I}$. Finite groups and compact connected semi simple Lie groups are such examples. In these cases the factor $\chi$ is absent in the representation of an infinitely divisible positive definite function.

For a compact connected group without infinitely divisible homomorphisms into $\mathscr{I}$ every normalised infinitely divisible positive definite function is of the form $\exp [\gamma(g)-\gamma(e)]$ where $\gamma$ is positive definite.

Remark 2. For any representation $T$ with cyclic vector $x$ in a Hilbert space $\mathscr{H}$ we can construct an infinitely divisible representation as follows: let $e(\mathscr{H})$ be the Hilbert space $\tilde{C} \oplus \mathscr{H} \oplus \mathscr{H} \otimes \mathscr{H} \oplus \cdots \oplus \underbrace{\mathscr{H} \otimes \cdots \otimes \mathscr{H}} \oplus \ldots$ and $e(x)$ be the vector

$\eta$ factors

$$
e^{-\frac{1}{2}\|x\| 2}\left\{1 \oplus x \oplus \frac{1}{\sqrt{2 !}}(x \otimes x) \oplus \cdots \oplus \frac{1}{\sqrt{n !}}(x \otimes \cdots \otimes x)+\ldots\right\} .
$$

Let $e(T)$ denote the restriction of the representation $1 \oplus T \oplus T \otimes T \oplus \ldots$ to the cyclic subspace generated by $e(x)$. Then $e(T)$ is an infinitely divisible represenation with cyclic vector $e(x)$.

If $U$ is an infinitely divisible representation with unit cyclic vector $x$ such that $\left(U_{g} x, x\right) \neq 0$ for any $g$ and $\left(U_{g} x, x\right)=\phi_{n}(g)^{n}$ for all $g \in G$ and $n=1,2, \ldots$ where $\phi_{n}$ is a sequence of positive definite functions converging to unity as $n \rightarrow \infty$, then $U$ is equivalent to $e(T)$ for some representation $T$. In the case of compact connected semisimple Lie groups every infinitely divisible representation $U$ is equivalent to some $e(T)$. 


\title{
References
}

1. Parthasarathy, K. R.: Probability measures on metric spaces. New York: Academic Press 1967.

2. Pontrjagin, L. S.: Topological groups. Princeton University Press 1946 (translated from Russian).

3. Streater, R. F.: A continuum analogue of the lattice gas. Commun. Math. Phys. 12, 226-232 (1969).

\author{
K. R. Parthasarathy \\ University of Manchester \\ Statistical Laboratory \\ Department of Mathematics \\ Manchester, M 139 PL/England
}

